\title{
Reliability of Friedewald formula in patients with type 2 diabetes mellitus and its relation to lipid profile in diabetes regulation
}

\author{
Didem Barlak Keti*, Sabahattin Muhtaroglu \\ Medical Biochemistry, Erciyes University, Turkey
}

\begin{abstract}
Introduction: Many laboratories utilize Friedewald formula $(F F)$ to analyze LDL cholesterol levels of patients including diabetes mellitus (DM). Therefore, it is essential to consider the coherence of results acquired by FF and direct measurement. The number of studies that investigated the effect of lipid parameters, especially TG/ $H D L$ cholesterol ratio, on the difference between the two methods is limited. The study was designed to compare LDL cholesterol values obtained by using FF with direct measurement, and to evaluate the relationship between diabetes regulation and lipid profile. Material and Methods: In the cross-sectional study, 529 type 2 DM patients and 1703 non-DM subjects were divided into four groups regarding TG concentrations. Unlike other studies, the study focuses on direct $L D L(D L D L)$ cholesterol levels obtained with the help of different DLDL cholesterol kits $(n=20)$. The correlations were implemented between HbAlc and lipid profiles. Results: It was determined that the bias $\%$ was over $10 \%$ in $24 \%$ of patients with 100-199 mg/dL TG levels. The parameter revealed that the most significant difference and the strongest correlation with HbAlc was TG/HDL cholesterol ratio in patients with type 2 DM. Conclusions: In patients with type 2 DM, even if it was $T G<200 \mathrm{mg} / \mathrm{dL}, \mathrm{LDL}$ calculated with FF should be evaluated together with the TG/HDL cholesterol ratio. Otherwise, direct measurement can be recommended. This ratio is related to diabetes regulation and may be used to monitor patients..
\end{abstract}

Keywords: Friedewald formula, LDL cholesterol, type 2 diabetes mellitus Received: 26 ${ }^{\text {th }}$ August 2020; Accepted: 15 th December 2020; Published: $28^{\text {th }}$ January 2021

\section{Introduction}

Cardiovascular diseases (CVD) remain a main reason of morbidity and mortality. Clinical practice guidelines advise low density lipoprotein (LDL) cholesterol as the fundamental lipid marker for risk estimation and management of coronary artery disease (CAD) $(1,2)$. Hence, the accurate measurement or estimation of LDL cholesterol is extremely significant for reliable evaluation of CVD risk (3). The reference method to analyze LDL cholesterol is time-consuming and requires costly equipments and trained

\footnotetext{
* Corresponding author: Didem Barlak Keti, Medical Biochemistry, Erciyes University, Turkey E-mail: dbarlakketi@erciyes.edu.tr
} 
personnel. For that reason, the most commonly utilized method for detecting LDL cholesterol concentration is the Friedewald formula (FF). However, this formula has some restrictions including fasting condition, individuals with triglyceride (TG) levels above $400 \mathrm{mg} / \mathrm{dL}$ and type III hyperlipoproteinemia incidents (4). Research suggested utilization of either direct LDL or apo B levels in patients with diabetes mellitus (DM), CVD and other high risk groups with TG levels $>200 \mathrm{mg} / \mathrm{dL}$ and LDL cholesterol $<70 \mathrm{mg} / \mathrm{dL}$ estimated by FF $(5,6)$.

It was shown that correctness of FF decreases as the level of TG increases (7). Moreover, the accuracy of FF was suspicious in some patient groups such as DM (8). Diabetes-related dyslipidemia is generally represented as an increment in TG and a reduction in high-density lipoprotein (HDL) cholesterol levels with a predominance of small, dense LDL (sdLDL) particles relatively normal LDL cholesterol values. Unlike LDL, nonHDL cholesterol can be considered as a total of all atherogenic apolipoprotein B including lipoproteins. Therefore, high nonHDL cholesterol shows increased risk of CAD $(9,10)$.

The ratio of TG to HDL cholesterol is a determiner of LDL particle size in patients with type 2 DM (11) and the increase in this ratio is the most powerful predictor of CAD among all the lipid parameters investigated (12).

It is important to reveal the correlation between diabetes regulation and dyslipidemia in order to give an idea about what lipid parameters will be useful in the follow-up of these patients. The number of studies that investigated the effect of lipid parameters, especially TG/HDL cholesterol ratio and nonHDL cholesterol, on the difference between calculated and directly measured LDL cholesterol are limited. Therefore, in the present study, it was aimed to compare LDL cholesterol values calculated by the FF with direct measurement at distinct TG levels in patients with type
$2 \mathrm{DM}$, to investigate lipid parameters that can be used instead of calculated LDL cholesterol, and to evaluate the relationship between diabetes regulation and lipid profile.

\section{Material and methods}

\section{Study population}

In cross-sectional study, the lipid parameters were assessed on 690 patients consecutively with type 2 DM and 2236 non-DM subjects visiting the University of Erciyes, Medical Faculty Hospital during October-December 2018. The study was approved by the ethics committee of the Medical Faculty of University of Erciyes (2018/503) and was conducted in accordance with the guidelines of the Declaration of Helsinki (as revised in 2013).

For lipid analyses, fasting blood samples were collected from subjects aged 18-85 years in tubes without anticoagulant. Blood specimens were centrifuged at $2000 \mathrm{~g}$ for 10 minutes. The measurements were completed within $8 \mathrm{~h}$ of specimen collection.

Specimens with TG $>400 \mathrm{mg} / \mathrm{dL}$, samples from participants whose fasting status was unknown and samples with lacking values of any of the lipid measurements were excluded from the study (Figure 1). The remaining 529 type 2 DM patients and 1703 non-DM subject samples were divided into four groups considering TG concentration ranges as follows: $<100,100-199,200$ 299 and 300-400 mg/dL.

Type 2 DM patients were diagnosed by the American Diabetic Association (ADA) 2015 criteria or those who had a previous diabetic history. Subjects with blood glucose level $<100 \mathrm{mg} /$ dL were accepted as non-DM. Informed consent was obtained from all individuals included in this study.

Patients with type 2 diabetes were divided into two groups as HbA1c $\leq 7$ and $>7$. 


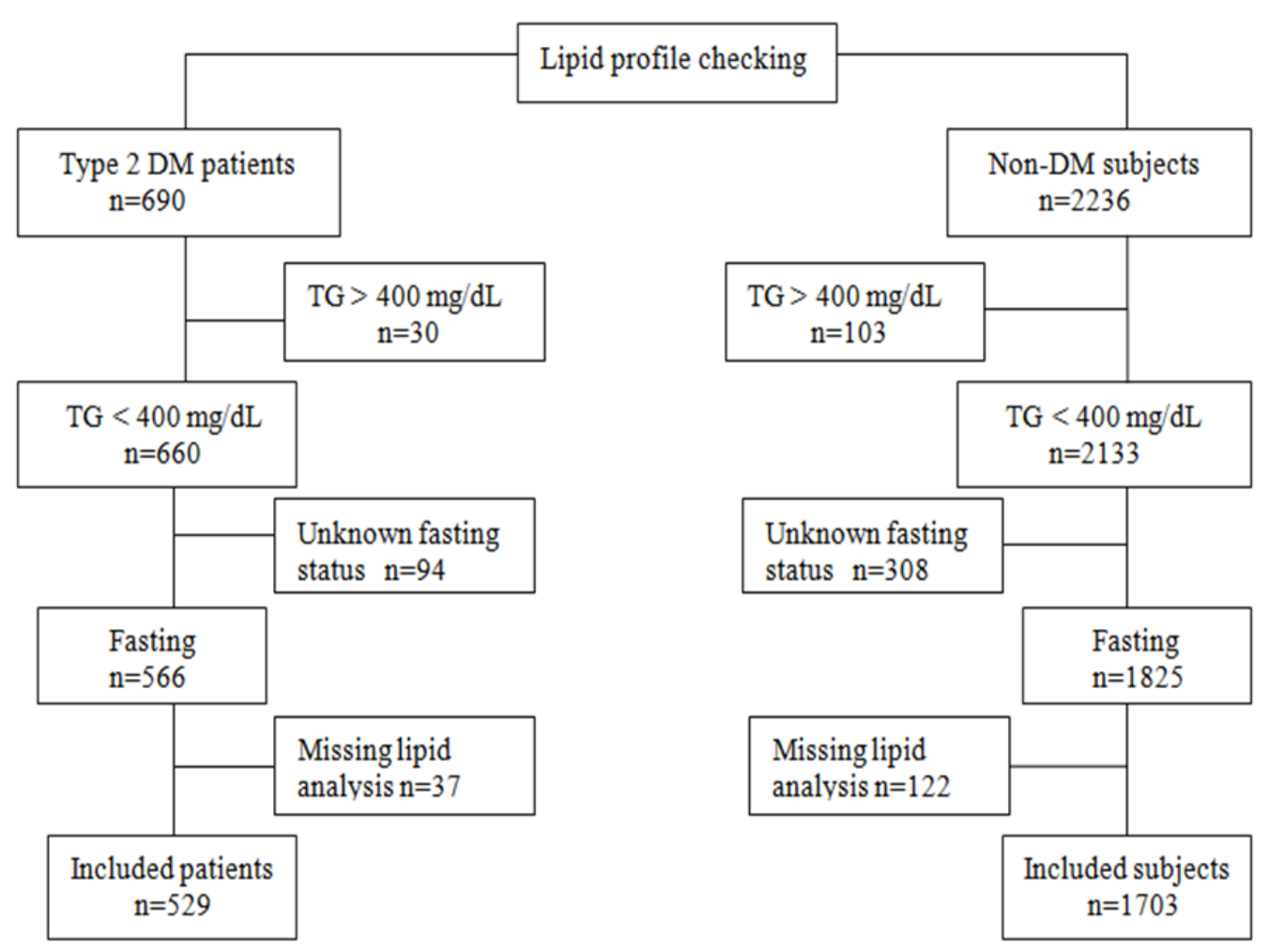

Fig. 1. Scheme showing the control of the subjects according to the inclusion criteria

\section{Biochemical analysis}

LDL cholesterol values were obtained from levels of total cholesterol, HDL cholesterol and TG up to $400 \mathrm{mg} / \mathrm{dL}$ using the FF. In this sudy, LDL (FLDL) cholesterol levels were calculated by using FF (FLDL cholesterol $=$ Total cholesterol - (TG/5+HDL cholesterol)), and the serum LDL cholesterol level was measured by using direct homogeneous assay. In order to show that the difference between LDL cholesterol levels calculated by using FF and those by the direct method is not method-based, direct LDL (DLDL) cholesterol levels were analyzed by using different DLDL cholesterol kits in alternative biochemical analyzers $(\mathrm{n}=20)$ in distinct TG levels. The direct assay utilized in the laboratory was indicated to provide current NCEP criteria for precision (coefficient of variation $<4 \%$ ), accuracy (bias $<4 \%$ ) and for total analytical error $(<12 \%)(5)$. Intra-as- say coefficient of variation for DLDL cholesterol was $0.7 \%$ at $116 \mathrm{mg} / \mathrm{dL}$ and the between-assay coefficient of variation was $2.6 \%$ at $100 \mathrm{mg} / \mathrm{dL}$. This method has been standardized against the beta quantification method as described in the advice of the LDL Cholesterol Method Certification Protocol for Manufacturers (13).

Serum total cholesterol and TG were measured by using enzymatic method. In addition, serum HDL cholesterol was determined with the help of direct homogeneous assay. All biochemical lipid analyses were performed on Cobas c702 chemistry autoanalyzer (Roche Diagnostics $\mathrm{GmbH}$, Mannheim, Germany). The nonHDL cholesterol value was computed by using the formula as total cholesterol minus HDL cholesterol. HbA1c levels were measured on Cobas c501 chemistry autoanalyzer (Roche Diagnostics GmbH, Mannheim, Germany). 


\section{Statistical Analyses}

The histogram and Q-Q plot were utilised to detect whether the data were normally distributed. The summarized statistics of normally and non-normally distributed continuous variables were exhibited as mean \pm standard deviation and median (25th/75th percentile), respectively. Summarized statistics of categorical variables were represented as frequency and percentage. The Mann-Whitney U test and independent sample $t$ test were applied to compare two groups. The Spearman test was utilized for correlation analysis. $\mathrm{P}$ value that is less than 0.05 was considered statistically significant.

\section{Results}

Patients with type $2 \mathrm{DM}$ were $56.98 \pm 11.44$ years of age while $213(40.0 \%)$ of them were men and $316(60.0 \%)$ women. Although there were not any significant differences in FLDL cholesterol levels; TG, total cholesterol, HDL cholesterol, nonHDL cholesterol, DLDL cholesterol, bias $\%$ and TG/HDL cholesterol values showed a statistical difference in patients with DM compared to those of non-DM subjects (Table 1).

As demonstrated in Table 2, the present study showed a statistically significant increase in TG,
TG/HDL cholesterol ratio, and a decline in HDL cholesterol, in patients with $\mathrm{HbA} 1 \mathrm{c}>7$. Although bias $\%$ and nonHDL cholesterol values were increased in patients with $\mathrm{HbA} 1 \mathrm{c}>7$ compared to those of patients with $\mathrm{HbA} 1 \mathrm{c} \leq 7$, no statistically significant difference was determined.

LDL cholesterol levels computed with FF compared to DLDL cholesterol levels in subjects with different TG values indicated the accuracy of the data that FF declines as TG increases. Bias\% between FLDL and DLDL cholesterol at $<100,100-199,200-299$ and 300-400 mg/dL TG levels were determined as $-5.7,-9,-13.3$ and -19.4 , respectively in patients with type $2 \mathrm{DM}$ (Table 3).

DLDL cholesterol levels of samples with different TG levels were assayed by using different DLDL cholesterol kits through an alternative biochemical analyzer, but a statistically significant difference was not detected.

A strong, significant, and positive correlation was found between $\mathrm{HbA} 1 \mathrm{c}$ and TG, HDL cholesterol, TG/HDL cholesterol ratio (Table 4).

In addition, it was shown in the study that there was a positive correlation between bias $\%$ and TG $(p<0.001)$ and TG/HDL cholesterol ratio $(p<0.001)$. Furthermore, although TG/HDL cho-

Table 1. The lipid profile in patients with type 2 DM and non-DM subjects

\begin{tabular}{|c|c|c|c|}
\hline Variables & $\begin{array}{l}\text { Patients with type 2DM } \\
n=529\end{array}$ & $\begin{array}{c}\text { non-DM subjects } \\
n=1703\end{array}$ & $\mathbf{p}$ \\
\hline Age (year) & $56.98 \pm 11.44$ & $55.87 \pm 11.77$ & 0.057 \\
\hline Sex (men/ women) & $213(40.0) / 316(60.0)$ & $675(39.6) / 1028(60.4)$ & 0.964 \\
\hline $\mathrm{TG}(\mathrm{mg} / \mathrm{dL})$ & $137.7(101 / 190.5)$ & $116.0(83.0 / 165.0)$ & $<0.001$ \\
\hline Total cholesterol $(\mathrm{mg} / \mathrm{dL})$ & $199.0(168.0 / 228.3)$ & $192.8(163.0 / 222.6)$ & 0.012 \\
\hline HDL cholesterol (mg/dL) & $48.6(41.0 / 57.0)$ & $50.0(41.0 / 60.8)$ & 0.021 \\
\hline FLDL cholesterol (mg/dL) & $116.3(93.6 / 143.5)$ & $113.2(88.7 / 140.8)$ & 0.058 \\
\hline DLDL cholesterol (mg/dL) & $130.0(106.0 / 157.0)$ & $125.0(99.0 / 152.0)$ & 0.004 \\
\hline Bias\% & $-9.2(-14.27 /-5.35)$ & $-8.14(-12.31 /-4.87)$ & $<0.001$ \\
\hline nonHDL cholesterol (mg/dL) & $146.9(119.9 / 178.1)$ & $140.0(111.0 / 170.5)$ & $<0.001$ \\
\hline TG/HDL cholesterol & $2.7(1.9 / 4.2)$ & $2.3(1.4 / 3.7)$ & $<0.001$ \\
\hline HbA1c (\%) & $7.1(6.4 / 8.3)$ & - & \\
\hline
\end{tabular}

Data expressed as median (25th/75th percentile), $\mathrm{n}(\%)$ and mean $\pm \mathrm{sd}$, DLDLcholesterol: direct measured LDLcholesterol; FLDL cholesterol: calculated LDL cholesterol by Friedewald formula 
Table 2. Comparison of lipid profile parameters at different HbA1c levels

\begin{tabular}{lccc}
\hline Variables & HbA1c $\leq \mathbf{7} \% \mathbf{~}(\mathbf{n}=\mathbf{2 5 5})$ & HbA1c $>$ 7 $\% \mathbf{~}(\mathbf{n}=\mathbf{2 7 4})$ & $\mathbf{p}$ \\
\hline TG & $129(98 / 178)$ & $146.90(105.52 / 197.25)$ & 0.011 \\
\hline Total cholesterol $(\mathrm{mg} / \mathrm{dL})$ & $199(170 / 230)$ & $198.60(165.72 / 226.25)$ & 0.761 \\
\hline HDL cholesterol $(\mathrm{mg} / \mathrm{dL})$ & $51(41 / 59)$ & $47.0(40-55.00)$ & 0.036 \\
\hline FLDL cholesterol $(\mathrm{mg} / \mathrm{dL})$ & $118.80(95.84 / 145)$ & $114.20(91.10 / 142.68)$ & 0.470 \\
\hline DLDL cholesterol $(\mathrm{mg} / \mathrm{dL})$ & $133(108 / 158)$ & $127(104 / 156)$ & 0.499 \\
\hline Bias\% & $-8.87(-13.82 /-5.36)$ & $-9.73(-14.58 /-5.31)$ & 0.332 \\
\hline nonHDL cholesterol $(\mathrm{mg} / \mathrm{dL})$ & $146.90(121.80 / 178.20)$ & $147.00(119 / 177.37)$ & 0.864 \\
\hline TG/HDL cholesterol & $2.54(1.78 / 4.00)$ & $3.12(2.04 / 4.54)$ & 0.006 \\
\hline HbA1c $(\%)$ & $6.3(5.9 / 6.7)$ & $8.2(7.6 / 9.6)$ & $<0.001$ \\
\hline
\end{tabular}

Data expressed as median (25th/75thpercentile)

Table 3. Comparison of FLDL cholesterol to DLDL cholesterol in patients with type 2 DM and non-DM at different triglyceride levels

\begin{tabular}{lcccc}
\hline Variables & Type 2 DM & Bias\% (Type 2 DM) & non-DM & Bias\% (non-DM) \\
\hline TG $<100 \mathrm{mg} / \mathrm{dL}$ & $\mathbf{( n = 1 2 7 )}$ & $\mathbf{( n = 6 5 3 )}$ \\
\hline DLDL cholesterol & $111.0(95.0 / 136.0)$ & $107.0(86.5-131.0)$ \\
\hline FLDL cholesterol & $107.6(86.0-130.3)$ & $-5.7(-10.1 /-2.6)$ & $100.2(80.2 / 123.9)$ & $-6.1(-9.3 /-3.1)$ \\
\hline TG 100-199 $\mathrm{mg} / \mathrm{dL}$ & $\mathbf{( n = 2 9 0 )}$ & $\mathbf{( n = 7 9 5 )}$ \\
\hline DLDL cholesterol & $131.0(108.0 / 156.5)$ & $132.6(108.0 / 159.0)$ \\
\hline FLDL cholesterol & $119.5(97.1 / 144.2)$ & $-9.0(-13.0 /-5.7)$ & $122.0(96.6 / 148.2)$ & $-8.4(-12.0 /-5.3)$ \\
\hline TG 200-299 $\mathrm{mg} / \mathrm{dL}$ & $\mathbf{( n = 9 1 )}$ & $\mathbf{( n = 2 0 5 )}$ \\
\hline DLDL cholesterol & $146.0(117.0 / 183.0)$ & $149.0(119.0 / 184.0)$ \\
\hline FLDL cholesterol & $127.8(99.0 / 160.0)$ & $-13.3(-18.3 /-8.8)$ & $128.2(98.3 / 161.4)$ & $-13.4(-17.9 /-9.9)$ \\
\hline TG 300-400 $\mathrm{mg} / \mathrm{dL}$ & $\mathbf{( n = 2 1 )}$ & $\mathbf{( n = 5 0 )}$ \\
\hline DLDL cholesterol & $159.0(122.5 / 177.5)$ & $144.9(117.5 / 171.8)$ \\
\hline FLDL cholesterol & $129.3(91.0 / 146.0)$ & $-19.4(-27.0 /-18.1)$ & $119.7(86.0 / 143.0)$ & $-18.7(-24.4 /-13.6)$ \\
\hline
\end{tabular}

Data expressed as median (25th/75th), \% Bias [(FLDL cholesterol) - (DLDL cholesterol) / (DLDL cholesterol)]*100

DLDL cholesterol $=$ Direct measured LDL cholesterol; FLDL cholesterol $=$ calculated LDL cholesterol by Friedewald formula

Table 4. Correlation of HbA1c to lipid profile in all patients with type 2 DM

\begin{tabular}{lc}
\hline Variables & $\begin{array}{c}\text { HbA1c } \\
\mathbf{p}(\mathbf{r h o})\end{array}$ \\
\hline TG & $0.003(0.130)$ \\
\hline Total cholesterol & $0.597(-0.023)$ \\
\hline FLDL cholesterol & $0.320(-0.043)$ \\
\hline DLDL cholesterol & $0.374(-0.039)$ \\
\hline HDL cholesterol & $0.003(-0.131)$ \\
\hline TG/HDL cholesterol & $<0.001(0.151)$ \\
\hline nonHDL cholesterol & $0.858(0.008)$ \\
\hline
\end{tabular}

lesterol ratio demonstrated a correlation with DLDLcholesterol $(\mathrm{p}<0.001)$, it did not have a correlation with FLDL cholesterol $(p=0.051)$ levels of all patients with type $2 \mathrm{DM}$.

The percentage of misclassified in risk categories for patients with DM by FF was $12.1 \%$ at the $100 \mathrm{mg} / \mathrm{dL}$ cut-off points (Direct measured LDL cholesterol was considered as the reference method).

\section{Discussion}

The FF has been commonly used to detect LDL cholesterol in routine laboratories since 1972 (4). The advantages of FF are its simple use, cost-effectiveness and availability in different 
populations. However, previous studies have shown that LDL cholesterol levels calculated by FF were different when compared to levels obtained by using direct assay $(14,15)$.

Anwar et al. (16) showed that FF overestimated LDL cholesterol at lower TG levels, and underestimated at elevated TG levels compared to that of the direct measurement. Lee et al. (17) found similar results. Many studies have proposed that the FF underestimates LDL cholesterol more than direct assay $(3,18,19)$.

Esawya et al. (20) observed that the difference between FLDL and DLDL cholesterol was higher in patients with DM compared to non-DM subjects. In other studies, similar results were obtained (7,21). In addition, the negative bias between FLDL and DLDL cholesterol was noted even at desirable TG levels (8).

Since many laboratories utilize FF to report LDL cholesterol for patients including DM (22), it is essential to consider the concordance of results obtained by FF and direct measurements.

In the present study, the negative bias\% values for FLDL and DLDL cholesterol were 13.3 and $19.6 \mathrm{mg} / \mathrm{dL}$ at 200 to $299 \mathrm{mg} / \mathrm{dL}$ and 300 to 400 $\mathrm{mg} / \mathrm{dL}$ TG levels, respectively in patients with type $2 \mathrm{DM}$. We determined that the negative bias \% was over $10 \%$ in $24 \%$ of patients with 100 to $199 \mathrm{mg} / \mathrm{dL}$ TG levels.

In dyslipidemic patients, although LDL cholesterol is the main target of treatment, current guidelines consider nonHDL cholesterol as the secondary goal in subjects with high TG (over $200 \mathrm{mg} / \mathrm{dL}$ ), the majority of whom are diabetics. Furthermore, nonHDL cholesterol was still an indicator of CAD in nonfasting individuals $(10,23)$. In addition, high nonHDL cholesterol, apo B, and DLDL cholesterol generally reflect increased concentrations of sdLDL particles that predominate when TG is high and HDL cholesterol is low (24).

In the current study, although FLDL cholesterol did not show a significant difference, DLDL cho- lesterol values were higher in patients with type 2 DM compared to those of non-DM subjects.

The LDL particles may be small and the risk of CAD may be increased in patients with type 2 $\mathrm{DM}$, even if the HDL cholesterol level is normal and TG level is $<200 \mathrm{mg} / \mathrm{dL}$. A TG/HDL cholesterol ratio that is $>3(\mathrm{mg} / \mathrm{dL})$ distinguishes the small and large LDL size pattern (11).

Our data showed that TG/HDL cholesterol ratio was $>3$ in $7.56 \%$ (40) of diabetic patients with normal HDL cholesterol levels (HDL $>40 \mathrm{mg} /$ $\mathrm{dL}$ men and $>50$ women) and $<200 \mathrm{mg} / \mathrm{dL}$ TG levels. In addition, when patients with DM were compared with those non-DM, the most significant statistical differences were in TG, nonHDL cholesterol values and TG/HDL cholesterol ratio. It is believed that nonHDL cholesterol and TG/HDL cholesterol should be reported in clinical laboratories for patients with type $2 \mathrm{DM}$ who have acceptable TG levels if LDL cholesterol is not measured directly.

The difference between DLDL and FLDL cholesterol was higher with the higher fasting glucose levels (25). Viera et al. (26) reported that this difference was higher in patients with $\mathrm{HbA} 1 \mathrm{c}$ $>8 \%$ than those with $\mathrm{HbA} 1 \mathrm{c}<8 \%$. However, in patients with DM, there was no significant difference between DLDL and FLDL cholesterol in different $\mathrm{HbA} 1 \mathrm{c}$ and HDL cholesterol groups. The TG level was the primary impact factor of correlation between the two methods (27).

Patients with $\mathrm{HbA} 1 \mathrm{c}$ value $>7.0 \%$ had significantly higher values of total cholesterol, TG, LDL cholesterol, and nonHDL cholesterol compared to the patients with $\mathrm{HbAlc} \leq 7.0 \%$. However, there was no significant difference in value of HDL cholesterol between the two groups (28). Babic et al. (29) stated that TG/HDL cholesterol ratio was significantly higher in patients with $\mathrm{HbA} 1 \mathrm{c} \geq 7 \%$ compared to patients with $\mathrm{HbA} 1 \mathrm{c}<7 \%$.

The current study did not reveal any significant difference between DLDL and FLDL cholesterol 
in patients with $\mathrm{HbA} 1 \mathrm{c}>7 \%$ compared to those with $\mathrm{HbA} 1 \mathrm{c} \leq 7 \%$. This result may depend on the number of patients with high TG level and the difference of $\mathrm{HbA1c}$ values determined to form groups.

A more significant difference in TG/HDL cholesterol ratio in patients with $\mathrm{HbA1c}$ value $>7.0 \%$ was detected when compared to TG or HDL cholesterol alone in patients with $\mathrm{HbA} 1 \mathrm{c} \leq 7.0 \%$.

Mahato et al. (27) reported that HbA1c demonstrated positive and significant correlations with total cholesterol, FLDL cholesterol, HDL cholesterol and nonHDL cholesterol. Additionally, significant positive correlation was observed between TG/HDL cholesterol ratio and HbA1c (29,30).

In the present study, HbAlc showed the strongest correlation with TG/HDL cholesterol ratio within lipid parameters in patients with type 2 DM.

Usage the FF can lead to the misclassification of patients with LDL cholesterol values near threshold (100 mg/dL) (22). A previous study suggested that estimation of LDL cholesterol by FF may be incorrect for evaluation of CVD risk in patients with type $2 \mathrm{DM}$ and may not be proper for the management of dislipidemia in those patients $(8,31)$. In this previous study, the FF overestimated by $>10 \%$ the true LDL cholesterol concentration in $39 \%$ of patients with DM and underestimated the actual value in $13 \%$ of patients, with only $48 \%$ accuracy (31).

Chai Kheng et al. (8) reported that $26 \%$ of patients with DLDL cholesterol $\geq 100 \mathrm{mg} / \mathrm{dL}$ were classified as LDLcholesterol $<100 \mathrm{mg} / \mathrm{dL}$ by using FF.

Twelve percent of patients determined in LDL cholesterol $>100 \mathrm{mg} / \mathrm{dL}$ by direct measurement were calculated as $<100 \mathrm{mg} / \mathrm{dL}$ by using FF. When TG/HDL cholesterol ratio $>3$ was used, half of the patients $(6 \%)$, who were miscalculated, were found to be at risk.

\section{Limitations}

Access to detailed clinical data of patients such as receiving statin therapy, hormone replacement therapy and presence of hypertension was not possible. Direct measurement for LDL cholesterol was not tested against reference method ( $\beta$ Q method). In addition, sdLDL cholesterol levels or sdLDL particles could not be analyzed in the present study. If sdLDL particle levels could be determined, it could be clarified whether the TG/ HDL cholesterol ratio can be used instead of sdLDL.

\section{Conclusion}

Based on the abovementioned findings, it can be stated that in patients with type $2 \mathrm{DM}$, even if TG $<200$, the LDL cholesterol value calculated with FF should be evaluated together with the TG/HDL cholesterol ratio. This ratio is also related to diabetes regulation and it is believed that this ratio may be used to monitor patients with type $2 \mathrm{DM}$, and may be suitable for evaluating CAD risk.

\section{Abbreviations}

CVD - cardiovascular diseases

LDL - low density lipoprotein

CAD- coronary artery disease

FF - Friedewald formula

TG - triglyceride

DM - diabetes mellitus

HDL - high density lipoprotein

sdLDL - small, dense LDL

ADA - American Diabetic Association

FLDL- calculated LDL cholesterol levels by using $\mathrm{FF}$

DLDL - direct LDL

Authors' contribution

DBK-Conceptualization, Methodology, Formal analysis, Writing-original draft preparation, Visualization 


\section{SM -Conceptualization, Validation, Writing-re- view and editing}

All the authors have accepted responsibility for the entire content of this submitted manuscript and approved submission.

\section{Conflicts of Interest}

None declared.

\section{References}

1. Grundy SM, Stone NJ, Bailey AL, Beam C, Birtcher KK, Blumenthal RS. 2018 AHA/ACC/AACVPR/AAPA/ ABC/ACPM/ADA/AGS/APhA/ASPC/NLA/PCNA guideline on the management of blood cholesterol: a report of the American College of Cardiology/American Heart Association Task Force on clinical practice guidelines. Circulation. 2019 Jun;139(25):1082-143. DOI: 10.1161/CIR.0000000000000698

2. Catapano AL, Graham I, Backer GD, Wiklund O, Chapman MJ, Drexel H, et al. 2016 ESC/EAS guidelines for the management of dyslipidaemias. Eur Heart J. 2016 Aug;37 (39):2999-3058. DOI: 10.1093/eurheartj/ ehw272

3. Kapoor R, Chakraborty M, Singh NA. Leap above Friedewald formula for calculation of lowdensity lipoprotein cholesterol. J Lab Physicians. 2015 JanJun;7(1):11-6. DOI: 10.4103/0974-2727.154780

4. Friedewald WT, Levy RI, Fredrickson DS. Estimation of the concentration of low density lipoprotein cholesterol in plasma, without use of the preparative ultracentrifuge. Clin Chem. 1972 Jun;18(6):499-502. DOI: 10.1093/clinchem/18.6.499

5. Jialal I, Gounden V. Reporting LDL cholesterol levels in the era of intensive lipid management: a clarion call. Clin Chem Lab Med. 2017 Aug;55(10):1447-9. DOI: 10.1515/cclm-2017-0639

6. Esteban-Salan M, Aguilar-Doreste JA, Arranz-Pena ML, Juve-Cuxart S, Gich-Salarich I, Zapico-Muniz $\mathrm{E}$, et al. Multi-centric evaluation of the homogeneous LDL cholesterol plus assay: comparison with beta-quantification and Friedewald formula. J Clin Biochem. 2008 Nov;41(16-17):1402-9. DOI: 10.1016/j. clinbiochem.2008.07.014

7. Fawwad A, Sabir R, Riaz M, Moin H, Basit A. Measured versus calculated LDL cholesterol in subjects with type
2 diabetes. Pak J Med Sci. 2016 Jul-Aug;32(4):955-60. DOI: 10.12669/pjms.324.9896

8. Chai Kheng EY, Chee Fang S, Chang S, Kiat Mun SL, Su Chi L, Lee Ying Y, et al. Low-density lipoprotein cholesterol levels in adults with type 2 diabetes: an alternative equation for accurate estimation and improved cardiovascular risk classification. Diab Vasc Dis Res. 2014 Nov;11(6):431-9. DOI: 10.1177/1479164114547703

9. Jialal I, Singh G. Management of diabetic dyslipidemia: an update. World J Diabetes. 2019 May;10(5):280-90. DOI: 10.4239/wjd.v10.i5.280

10. Su X, Kong Y, Peng D. Evidence for changing lipid management strategy to focus on non-high density lipoprotein cholesterol. Lipids Health Dis. 2019 Jun;18(1):134. DOI: 10.1186/s12944-019-1080-x

11. Boizel R, Benhamou PY, Lardy B, Laporte F, Foulon T, Halimi S. Ratio of triglycerides to HDL cholesterol is an indicator of LDL particle size in patients with type 2 diabetes and normal HDL cholesterol levels. Diabetes Care. 2000 Nov;23(11):1679-85. DOI: 10.2337/diacare.23.11.1679

12. da Luz PL, Favarato D, Faria-Neto Junior JR, Lemos P, Chagas ACP. High ratio of triglycerides to HDL-cholesterol predicts extensive coronary disease. Clinics. 2008 Aug;63(4):427-32. DOI: 10.1590/S180759322008000400003

13. Cholesterol Reference Method Laboratory Network (CRMLN). National reference system for cholesterol. LDL cholesterol method certification protocol for manufacturers. 2006.1-13.

14. Mora S, Rifai S, Buring JE, Ridker PM. Comparison of LDL cholesterol concentrations by Friedewald calculation and direct measurement in relation to cardiovascular events in 27331 women. Clin Chem. 2009 May;55(5):888-94. DOI: 10.1373/ clinchem.2008.117929

15. Chatterjee BP, Sendhav S, Kakaiya A, Biswas PC. A comparative analysis of direct LDL-C assay and Friedewald's formula in subjects of ischemic heart disease \& stroke in a tertiary care centre. Int J Clin Biochem Res. 2018 July;5(4):541-6. DOI: 10.18231/23946377.2018 .0115

16. Anwar M, Khan DA, Khan FA. Comparison of Friedewald formula and modified Friedewald formula with direct homogeneous assay for low density lipoprotein cholesterol estimation. J Coll Physicians Surg Pak. 2014 Jan;24(1):8-12. 
17. LeeJ, Jang S, Jeong H, Ryu OH. Validation of the Friedewald formula for estimating low density lipoprotein cholesterol: the Korea National Health and Nutrition Examination Survey, 2009 to 2011. Korean J Intern Med. 2020 Jan;35(1):150-9. DOI: 10.3904/ kjim.2017.233

18. Martin SS, Blaha MJ, Elshazly MB, Brinton EA, Toth PP, McEvoy JW, et al. Friedewald-estimated versus directly measured low-density lipoprotein cholesterol and treatment implications. J Am Coll Cardiol. 2013 Aug;62(8):732-9. DOI: 10.1016/j.jacc.2013.01.079

19. Bansal E, Kaur N. Does Friedewald formula underestimate the risk of ischemic heart disease? Indian J Clin Biochem. 2014 Oct;29(4):496-500. DOI: 10.1007/ s12291-013-0392-2

20. Esawya MM, Shabanaa MA, Magdyb MM. Evaluation of Martin's equation for LDL-C estimation in type 2 diabetes mellitus Egyptian patients. Clin Chim Acta. 2019 Aug;495:487-92. DOI: 10.1016/j.cca.2019.05.025

21. Kurniawan LB, Windarwati W, Mulyono B. Analysis of LDL-C measurement using direct and Friedewald formula in type 2 diabetes mellitus patients. Indonesian J Clin Pathol Med Lab. 2018 July;24(3):255-7. DOI: 10.24293/ijcpml.v24i3.1339

22. Razi F, Forouzanfar K, Bandarian F, Nasli-Esfahani E. LDL-cholesterol measurement in diabetic type 2 patients: a comparison between direct assay and popular equations. J Diabetes Metab Disord. 2017 Nov;16:43. DOI: $10.1186 / \mathrm{s} 40200-017-0326-2$

23. Enkhmaa B, Prakash N, Berglund L. Non-HDL-C levels and residual cardiovascular risk: do population-specific precision approaches offer any advantages? Atherosclerosis. 2018 Jul;274:230-1. DOI: 10.1016/j.atherosclerosis.2018.05.010

24. Mora S, Buring JE, Ridker PM. Discordance of low-density lipoprotein (LDL) cholesterol with alternative LDL-related measures and future coronary events. Circulation. 2014 Feb;129(5):553-61. DOI: 10.1161/

\section{CIRCULATIONAHA.113.005873}

25. Sudha K, Ashok P, Kiran KAM, Aradhana M, Anupama H. Validation of the Friedewald formula in type II diabetes mellitus: an Indian perspective study. Int J Biomed Adv Res. 2015 Feb;6(2):103-6. DOI: 10.7439/ ijbar.v6i2.1581

26. Viera PL, Araujo GN, Telo GH, Smidt LFS, Jost MF, Furtado MV,et al. Low-density lipoprotein values estimated by Friedewald equation are affected by diabetes control. Int J Cardiovasc Sci. 2016 Aug;29(5):348-54. DOI: $10.5935 / 2359-4802.20160045$

27. Mahato RV, Gyawali P, Raut PP, Regmi P, Singh KP, Pandeya DR, et al. Association between glycaemic control and serum lipid profile in type 2 diabetic patients: glycated haemoglobin as a dual biomarker. Biomed Res. 2011 March;22(3):375-80.

28. Dong QT, Gao Y,Wu NQ, Guo YL, Zhu CG, Li S, et al. Impact of glucose and lipid markers on the correlation of calculated and enzymatic measured low-density lipoprotein cholesterol in diabetic patients with coronary artery disease. J Clin Lab Anal. 2018 Jun;32(5):e22399. DOI: $10.1002 /$ jcla.22399

29. Babic N, Valjevac A, Zaciragic A, Avdagic N, Zukic S, Hasic S. The triglyceride/HDL ratio and triglyceride glucose index as predictors of glycemic control in patients with diabetes mellitus type 2 . Med Arch. 2019 Jun;73(3):163-8. DOI: 10.5455/medarh.2019.73.163-168

30. Ozder A. Lipid profile abnormalities seen in T2 DM patients in primary Health care in Turkey: a cross-sectional study. Lipids Health Dis. 2014 Dec;13:183. DOI: 10.1186/1476-511X-13-183

31. Rubies-Prat J, Reverter JL, Sentí M, Pedro-Botet J, Salinas I, Lucas A, et al. Calculated low-density lipoprotein cholesterol should not be used for management of lipoprotein abnormalities in patients with diabetes mellitus. Diabetes Care. 1993 Aug;16(8):1081-6. DOI: 10.2337/diacare.16.8.1081 